\title{
Origin of the Kinetic Heterogeneity of Ultrafast Light-Induced Electron Transfer from Ru(II)-Complex Dyes to Nanocrystalline Semiconducting Particles
}

\author{
Bernard Wenger§, Michael Grätzel, and Jacques-E. Moser ${ }^{\star}$ \\ $\S$ SCS Poster Prize Winner
}

\begin{abstract}
Interfacial electron transfer from a molecular dye to a semiconductor is a keystone process in the conversion of light into electricity in dye-sensitized solar cells. The most successful devices developed so far are based on the sensitization of nanocrystalline titanium dioxide by ruthenium polypyridyl complexes. The ultrafast electron injection from the widely used $\mathrm{Ru}^{\prime \prime}(\mathrm{dcbpy})_{2}(\mathrm{NCS})_{2}$ dye in particular has been intensely studied. Several research groups, including ours, have found that this reaction apparently proceeds with a fast sub-100 fs phase, followed by a slower kinetic component with a time constant of $0.7-100$ ps and accounting for $16-65 \%$ of the total yield. No convincing explanation has been provided for a clear understanding of the origin of this non-exponential kinetic behavior. In this contribution we show that aggregation of dye molecules at the interface is actually responsible for the slow kinetic component of the interfacial electron transfer. A thorough control of the dissolution of the dye and of its adsorption onto nanocrystalline oxide films allowed the reduction of the portion of dye excited states that react within the slow compartment and even made the latter completely disappear. In the absence of dye aggregates, femtosecond pump-probe studies of the sensitizer's oxidized state appearance yielded a rate constant for charge injection $>5 \times 10^{13} \mathrm{~s}^{-1}$, corresponding to an electron transfer time of less than $20 \mathrm{fs}$.
\end{abstract}

Keywords: Charge transfer dynamics - Dye aggregation - Femtosecond transient absorbance spectroscopy . Light-induced interfacial electron transfer $\cdot$ Non-exponential kinetics

\section{Introduction}

Interfacial electron transfer at dye/semiconductor boundaries has been under active study for the last decades due to its importance in applications like photography, xerography, and more recently dye-sensitized solar cells (DSC). In all these technologies, the first essential step is constituted by a light-induced interfacial electron transfer from a dye sensitizer, generally anchored onto small semiconducting micro- or nanoparticles. A thorough understanding of the dynamics of such a process is required for a smart design of new devices. In dye-

${ }^{*}$ Correspondence: PD Dr. Jacques-E. Moser Laboratory for Photonics \& Interfaces

Institute of Chemical Sciences \& Engineering

Ecole Polytechnique Fédérale de Lausanne

EPFL-ISIC-LPI, Station 06

$\mathrm{CH}-1015$ Lausanne

Tel.: + 41216933628

Fax: + 41216934111

E-Mail: je.moser@epfl.ch

http://photochemistry.epfl.ch sensitized photovoltaic solar cells, conversion of light into electricity is achieved by absorption of visible light by a molecular dye sensitizer ( $\mathrm{S}$ ) anchored on a large bandgap semiconductor (SC) [1]. An electron is transferred from the excited state of the dye to the conduction band of the semiconductor (Eqn. 1) and then diffuses in the solid until it reaches the external electrical circuit.

$$
\begin{aligned}
& \mathrm{SISC}+\mathrm{h} v \rightarrow \mathrm{S}^{*}\left|\mathrm{SC} \rightarrow \mathrm{S}^{+}\right| \mathrm{SC}+\mathrm{e}^{-} \mathrm{cb}^{(\mathrm{SC})}(1) \\
& \mathrm{S}^{+} \mid \mathrm{SC}+\mathrm{D} \rightarrow \mathrm{SISC}+\mathrm{D}^{+}
\end{aligned}
$$

Sustainable charge separation is obtained by using a redox relay (D) in solution (usually the iodide-triiodide couple), whose role is to quickly reduce the oxidized state of the dye sensitizer at the interface (Eqn. 2 ). The electrolyte also ensures the transport of positive charges by diffusion of oxidized relay species to a counter electrode. The photon-to-current conversion efficiency in this type of solar cell is controlled by the kinetic competition between several processes. The charge injection step has long been believed to compete only with the radiative and non- radiative deactivation of the excited dye. Since the lifetime of the excited states of efficient sensitizers in solution is typically of the order of hundreds of picoseconds to nanoseconds, it has been inferred that ultrafast interfacial electron transfer should always be characterized by a unit quantum yield and that sub-ps charge injection is kinetically redundant. The latest developments in the design of DSC involve the use of redox-active ionic liquids as the electrolyte [2]. In such media, where the concentration of the electron donor could be as high as $6 \mathrm{M}$, reductive quenching of the dye excited states by the redox relay (Eqn. 3) was found to take place within a few picoseconds [3]. Thus, the completion of the interfacial electron transfer process in the sub-100 fs time frame actually becomes a crucial requirement to ensure charge injection takes place with a high efficiency.

$\mathrm{S}^{*}\left|\mathrm{SC}+\mathrm{D} \rightarrow \mathrm{S}^{-}\right| \mathrm{SC}+\mathrm{D}^{+}$

So far most efficient DSC are based on the sensitization of titanium dioxide nanocrystalline films by ruthenium polypyridyl 
complexes [1]. The rate of the electron injection into $\mathrm{TiO}_{2}$ for these compounds was found to occur in the fs/ps time domain [4][5]. The conjunction of interest for solar cells development and fundamental understanding of ultrafast charge transfer has encouraged many groups to study the parameters influencing the dynamics of the interfacial electron transfer from the excited dye to the continuum of acceptor states constituting the semiconductor's conduction band. The most popular candidate for efficient solar energy harvesting is a dye sensitizer known as N3 (Fig. 1) [6]. Since 1996, several kinetic studies involving this dye anchored on metal oxides like $\mathrm{TiO}_{2}, \mathrm{SnO}_{2}$, and $\mathrm{ZnO}$ have been achieved [4][7-9]. Most recent studies have reported a biphasic behavior for the rise of the dye cation $\left(\mathrm{S}^{+}\right)$appearing upon charge injection. Although these works all agree with a fast, sub-100 fs phase followed by a slower component in the ps time scale [7][8], discrepancies appear concerning the relative importance of these two kinetic compartments and the time constant for the second phase. The reported amplitudes for the slower part range from $16 \%$ to $65 \%$ and the time constants vary between $700 \mathrm{fs}$ and 100 ps with a marked non-exponentiality. Attempts to justify this behavior were made by considering charge injection from various electronic and vibronic states of the dye [9]. However, no conclusive evidence was obtained to explain the large kinetic heterogeneity of interfacial electron transfer observed in the particular case of $\mathrm{Ru}$ (II)-complex dye sensitizers and the strong dependence of measured dynamics upon experimental conditions [7][8].

In this contribution, ultrafast pump-probe laser spectroscopy was used to measure the kinetics of the formation of the dye oxidized state upon electron injection from the excited state into $\mathrm{TiO}_{2}$. Particular attention was paid to the way the dye solution was prepared and the sensitizer adsorbed onto the oxide surface. The slow component observed for electron transfer is shown to be directly related to dye aggregates and the actual charge transfer from the adsorbed monomeric sensitizer characterized by a single-exponential kinetics with a time constant shorter than $20 \mathrm{fs}$.

\section{Results and Discussion}

Transparent, $8 \mu \mathrm{m}$-thick nanocrystalline films of $\mathrm{TiO}_{2}$ were prepared following a method identical to that used for dye-sensitized solar cells [10]. Films were soaked overnight in the dye solution. N3 was adsorbed from an ethanolic solution and a doubly deprotonated form of this dye TBAN3 $\left(\left(\mathrm{Bu}_{4} \mathrm{~N}\right)_{2}\left[\mathrm{Ru}(\mathrm{dcbpyH})_{2}(\mathrm{NCS})_{2}\right]\right)$ from a solution in $\mathrm{CH}_{3} \mathrm{CN} /$ tert-BuOH (1:1) solvent mixture [11]. Anchoring of N3 and TBA-N3 sensitizer molecules is known to take place

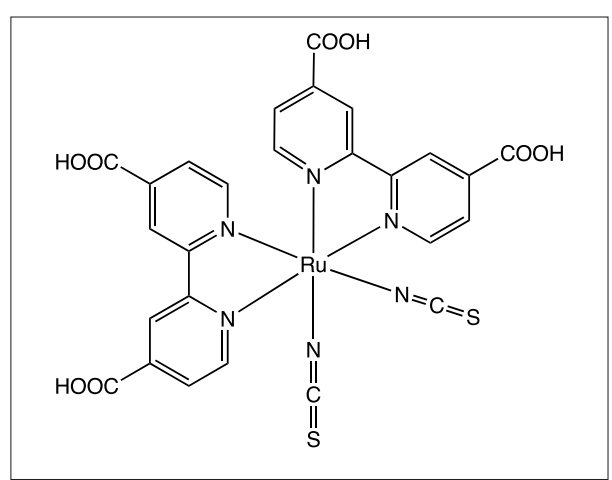

Fig. 1. Structure of the $\mathrm{Ru}^{\prime \prime}\left(\mathrm{dcbpyH}_{2}\right)_{2}(\mathrm{NCS})_{2}$ complex dye (N3).

through the two carboxylic groups that are in trans position to the -NCS ligands. For N3, this anchoring is associated with the deprotonation of the involved carboxylic groups. Therefore, species adsorbed on the $\mathrm{TiO}_{2}$ surface are expected to be identical for both forms of the dye. Films derivatized with N3 and TBA-N3 indeed displayed similar spectra with a broad maximum at $532 \mathrm{~nm}$, corresponding to a metal-to-ligand charge transfer transition (MLCT) of the complexes [12]. A redox inactive ionic liquid (1-ethyl-2-methylimidazolium bis(trifluoromethyl(sulfonyl) imide) was used as a reaction medium in all samples [13]. Ultrafast pump-probe spectroscopy allowed the direct observation of the dye cation formation resulting from the electron transfer to the semiconductor's conduction band. The setup employed was described previously [14]. It consisted of a Ti: sapphire amplified femtosecond laser system delivering $120 \mathrm{fs}$ pulses at $778 \mathrm{~nm}$. Two non-collinearly pumped optical parametric amplifiers (NOPA) were fed by the NIR output of the laser and were used to produce two independent wavelength-tunable beams. After compression in prism pairs, resulting pulses were characterized by a relatively narrow spectral width and a typical duration of $30 \mathrm{fs}$. In the pump-probe scheme used in this experiment the excitation beam was set at a wavelength of $535 \mathrm{~nm}$. This beam was focused on the sample that was continuously rotated to avoid degradation of the dye. The probe beam was tuned at a wavelength of $860 \mathrm{~nm}$, where the contribution of the oxidized dye absorption is maximized with respect to that of the excited state. Pulses traveled along an optical delay line before passing through the transparent sample and being detected.

To allow for a direct comparison of results with data reported in a majority of earlier studies, a commercially available N3 dye (Solaronix, Switzerland) was chosen in the first place. The sensitizer was adsorbed on $\mathrm{TiO}_{2}$ films from $3 \times 10^{-4} \mathrm{M}$ ethanolic solutions and dyed samples were covered by a film of the ionic liquid. The time evolution of the formation of the oxidized dye following ultrashort laser pulse excitation was measured. As depicted in Fig. 2 (trace a and insert), the absorption change due to the dye oxidized state was observed to rise with a first sub-100 fs phase followed by a slower kinetic component. Data were fitted with a sum of two analytical convolutions of a Gaussian instrument response (cross-correlation time of $c a .57 \mathrm{fs}$ ) with exponential growths with $\tau_{1}=76$ fs $(84 \%)$ and $\tau_{2}=45 \mathrm{ps}(16 \%)$. In comparison with available data from the literature, the amplitude of the second kinetic compartment was found to be rather small [8]. However, the reproducibility of this amplitude was poor. Depending on the film preparation (adsorption time, dye solution concentration, ...), relative amplitudes varied considerably, while kinetics were found to remain practically identical. This study was extended to the use of home-synthesized N3 and TBAN3. The observed amplitude of the slow part was considerably reduced by using our own preparation of N3 (data not shown) and disappeared totally when the deprotonated variant of the dye was used (Fig. 2, trace b).

The variability of the amplitude of the ps phase and, furthermore, the total disappearance of this component for TBA-N3 indicate that the origin of the biphasic kinetic behavior is not to be found in the photophysical properties of the dyes but rather in their colligative properties. Indeed, resonance Raman spectroscopy applied to dye solutions and derivatized oxide films showed that $\mathrm{H}$ bonded aggregates of the Ru-complex dyes pre-exist in ethanolic medium and are also observed on the surface [3b]. Contrary to the cases of dye molecules such as cynanines or xanthenes, aggregation does not cause any observable change in the absorption spectrum of N3 and TBA-N3. Aggregation of the dye on the surface provides an obvious rationale for the kinetic heterogeneity of interfacial electron transfer. Electron tunneling theory predicts an exponential dependence for electron transfer with the distance separating the donor and the acceptor. Assuming a dumping factor $\beta=1.2 \AA^{-1}$, the distance increase required to explain the observed slowing down of the injection could be estimated as 3-7 $\AA$. This figure is consistent with the geometric separation induced by dye aggregation. Diffusion of excitons within dye aggregates and intermolecular electron transfer mechanisms could also account for a spreading of interfacial reaction rates. The main difference between the N3 and the TBA-N3 form of the sensitizer lies in the solubility of these two compounds. TBA-N3 is more soluble in polar solvents and, because of electrostatic repulsion, dianions of the deprotonated dye are less prone to aggregate. The commercial N3 powder (Solaronix), constituted of large crystals, appeared to be harder to solubilize. Sonica- 


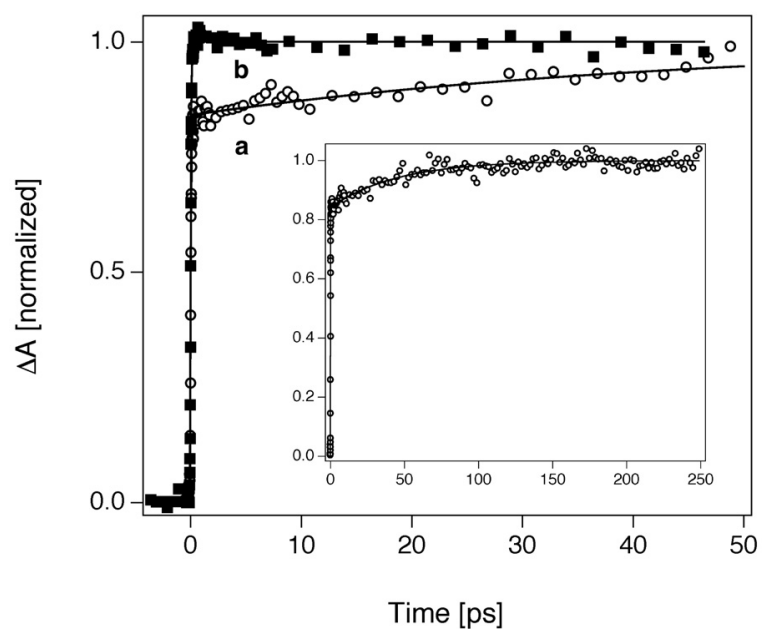

Fig. 2. Transient absorption signal obtained upon femtosecond pulse excitation of N3 (O) (Solaronix) and TBA-N3 ( $\square$ ) complex dyes adsorbed on nanocrystalline $\mathrm{TiO}_{2}$ films. The insert shows trace (a) on an extended time scale. Excitation pulse wavelength: $535 \mathrm{~nm}$, probe wavelength: 860 $\mathrm{nm}$. The observed growth of the signal corresponds to the appearance of the dye oxidized state produced by the charge injection process.

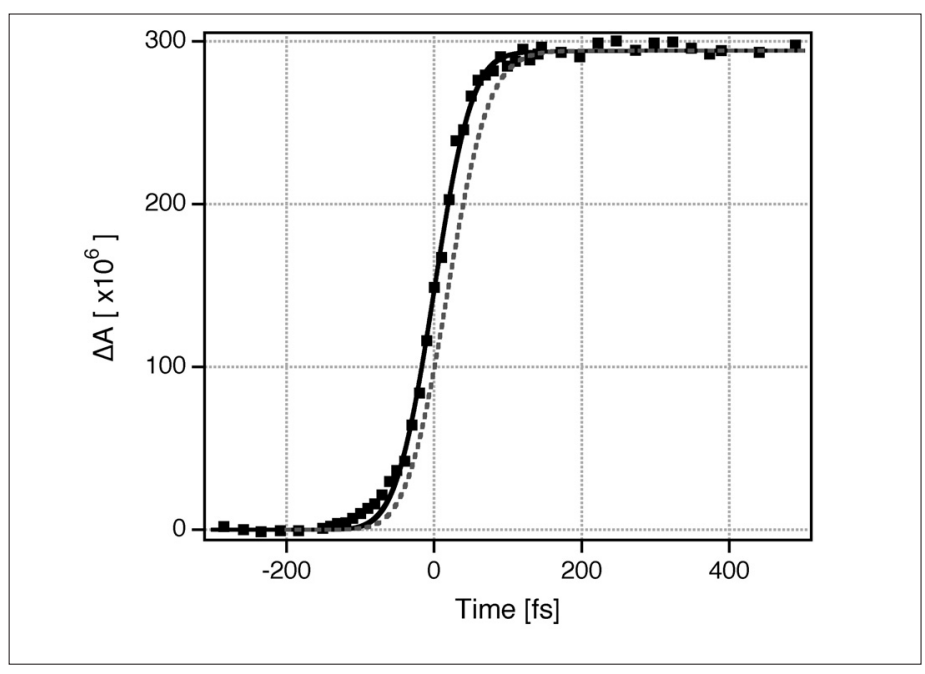

Fig. 3. Transient absorption signal obtained upon ultrafast laser excitation of a typical TBA-N3-derivatized $\mathrm{TiO}_{2}$ film. The dashed line represents an analytical convolution of the Gaussian instrument response with an exponential growth function with $\tau=20$ fs. tion of this compound in ethanol, as often reported in the literature, tend to produce a colloidal suspension of the dye, thus favoring the deposition of pre-existing aggregates onto the oxide surface. The poor reproducibility of the amplitude of the slow kinetic component in our experiments and in the literature is compatible with various degree of aggregation, depending on the adsorption time and history of the dye solution.

The complete disappearance of the slow phase in the case of TBA-N3 adsorbed from diluted solutions of the dye $(<0.4 \mathrm{mM})$ suggests that the sensitizer is adsorbed as a monomer on the surface. These conditions allow the determination of the electron injection rate freed of artifacts due to dye aggregation. Fig. 3 shows the time evolution of the transient absorption measured at $850 \mathrm{~nm}$ with a TBA-N3-dyed $\mathrm{TiO}_{2}$ film covered by the ionic liquid. An analytical convolution of a Gaussian instrument response with an exponential growth with $\tau=20 \mathrm{fs}$ showed that the charge injection process clearly takes place on a time shorter than this limit. The interfacial electron transfer rate constant is therefore estimated to be $>5 \times 10^{13} \mathrm{~s}^{-1}$. An electron transfer rate constant of this magnitude implies the electronic coupling of the excited dye with the quasi-continuum of acceptor states constituting the conduction band of the solid is quite strong. In the normal Marcus approach to model the dynamics of electron transfer processes, the rate determining step is constituted by the relaxation of intramolecular and solvent nuclear degrees of freedom. For most species the time required for this vibrational reorganization is limited to a lower value of $100 \mathrm{fs}$. In our case, electron transfer is significantly faster than typical nuclear relaxation and is believed to be only controlled by the electron dephasing time of the semiconductor.

\section{Conclusion}

In the present study we showed that the kinetic heterogeneity of electron injection from the widely used $\mathbf{N} 3$ dye to $\mathrm{TiO}_{2}$ was not due to the excited state dynamics of the complex as previously suggested but rather to the aggregation of the sensitizer on the semiconductor surface. The total disappearance of the slow kinetic compartment in the case of an adsorbed monomeric form of the dye allowed an upper limit for the electron transfer time constant of 20 fs to be estimated, beyond the Marcus description of vibrationally-coupled electron transfer.

\section{Acknowledgement}

We gratefully acknowledge Dr. M.K. Nazeeruddin for the preparation of N3 and TBA$\mathbf{N} 3$ complexes. This research was made possible thanks to the financial support of the Swiss National Science Foundation and a grant of the Ecole Polytechnique Fédérale de Lausanne.

Received: January 6, 2005

[1] M. Grätzel, Nature 2001, 414, 338; A. Hagfeldt, M. Grätzel, Acc. Chem. Res. 2000 , 33, 269.

[2] P. Wang, S.M. Zakeeruddin, J.E. Moser, M. Grätzel, J. Phys. Chem. B 2003, 107, 13280; P. Wang, S.M. Zakeeruddin, J.E. Moser, R. Humphry-Baker, M. Grätzel, $J$. Am. Chem. Soc. 2004, 126, 7164.

[3] a) P. Wang, B. Wenger, R. Humphry-Baker, J.E. Moser, J. Teuscher, W. Kantlehner, J. Mezger, E.V. Stoyanov, P. Liska, S.M. Zakeeruddin, M. Grätzel, J. Phys. Chem. B $\mathbf{2 0 0 5}$
109 (in press); b) B. Wenger, J. Teuscher, M. Grätzel, J.E. Moser, to be published.

[4] M. Grätzel, J.E. Moser, in 'Electron Transfer in Chemistry, Vol. 5: Molecular-level electronics, imaging and information, energy and the environment', Eds. V. Balzani, I.R. Gould, Wiley-VCH, Weinheim, 2001, pp 589-644 and references therein.

[5] Y. Tachibana, J.E. Moser, M. Grätzel, D.R. Klug, J.R. Durrant, J. Phys. Chem. 1996, 100, 20056.

[6] M.K. Nazeeruddin, A. Kay, I. Rodicio, R Humphry-Baker, E. Müller, P. Liska, N. Vlachopoulos, M. Grätzel, J. Am. Chem. Soc. 1993, 115, 6382.

[7] J.B. Asbury, N.A. Anderson, E.C. Hao, X. Ai, T.Q. Lian, J. Phys. Chem. B 2003, 107, 7376.

[8] J. Kallioinen, G. Benkö, P. Myllyperkiö, L. Khriachtchev, B. Skårman, R. Wallenberg, M. Tuomikoski, J. Korppi-Tommola, V. Sundström, A.P. Yartsev, J. Phys. Chem. B 2004, 108, 6365 .

[9] G. Benkö, J. Kallioinen, J. Korppi-Tommola, A.P. Yartsev, V. Sundström, J. Am. Chem. Soc. 2002, 124, 489.

[10] C.J. Barbe, F. Arendse, P. Comte, M. Jirousek, F. Lenzmann, V. Shklover, M. Grätzel, J. Am. Ceram. Soc. 1997, 80, 3157.

[11] M.K. Nazeeruddin, P. Pechy, T. Renouard, S.M. Zakeeruddin, R. Humphry-Baker, P. Comte, P. Liska, L. Cevey, E. Costa, V. Shklover, L. Spiccia, G.B. Deacon, C.A. Bignozzi, M. Grätzel, J. Am. Chem. Soc. 2001, 123, 1613.

[12] M.K. Nazeeruddin, R. Humphry-Baker, P. Liska, M. Grätzel, J. Phys. Chem. B 2003, 107, 8981.

[13] P. Bonhôte, A.P. Dias, N. Papageorgiou, K. Kalyanasundaram, M. Grätzel, Inorg. Chem. 1996, 35, 1168.

[14] S. Pelet, M. Grätzel, J.E. Moser, J. Phys. Chem. B 2003, 107, 3215. 